\title{
MR Image Segmentation Based on Contrast Enhancement with Collaborative Learning
}

\author{
${ }^{1}$ Yuchou Chang \\ ${ }^{1}$ Computer Science and Engineering Technology Department, University of Houston - Downtown, \\ Houston, United States \\ changy@uhd.edu
}

\begin{abstract}
Magnetic resonance imaging (MRI) has been widely used on clinical applications. Due to different weighting strategies MRI, different weighting images have different contrast even for the same anatomy structure of the same subject. Since some MR brain images have low contrast, different types of tissues such as white matter, grey matter, and cerebrospinal fluid (CSF) are difficult to be divided and segmented. Image contrast needs to be enhanced for better post-processing and image analysis. In this paper, a two-step MR brain image segmentation technique is proposed to solve low contrast MR image segmentation problem. A collaborative learning based image enhancement is firstly applied on low contrast MR brain image. Then, spectral clustering algorithm is used for segmenting enhanced image. Experimental results illustrate that the proposed 2-step segmentation method is able to identify boundaries between tissues well, so that MR image segmentation accuracy is improved in compared to image segmentation without contrast enhancement and exact histogram equalization enhanced image segmentation.
\end{abstract}

Keywords: MR Image Segmentation, Image Enhancement, Spectral Clustering, Histogram Equalization, and Collaborative Learning.

\section{Introduction}

Magnetic Resonance Imaging (MRI) [1] has become an important aided tool in the clinical medical analysis of brain tissue structure to analyze the visualized three-dimensional reconstruction of disease diagnosis and surgical treatment, because of its advantages of non-invasion, high resolution, and good imaging quality. Compared with other medical imaging methods, MR imaging has higher soft tissue resolution and approximate anatomical imaging display, which can reflect the biochemical characteristics of organs or tissues and is very suitable for human brain imaging. Image segmentation [2] is an important image analysis technology, which determines the final analysis quality. It is the technical process of dividing the image into regions with different features and extracting the target of interest. With the wide application of medical images in clinical diagnosis, image segmentation has played an increasingly important role in medical image analysis. It is also the prerequisite and key technology of medical image visualization, image fusion, pattern recognition and image guided surgery. It is also the basis of practical application of medical image processing system in clinic. Due to the complexity and diversity of medical images, no segmentation algorithm is suitable for all images. Brain magnetic resonance (MR) image segmentation, as the basis of brain image analysis, plays a guiding role in the research of brain tissue brain diseases. It is also difficult in the field of image

DOI: $10.14738 /$ aivp.61.4089

Publication Date: 03 ${ }^{\text {rd }}$ Feb, 2018

URL: http://dx.doi.org/10.14738/aivp.61.4089 
Yuchou Chang; MR Image Segmentation Based on Contrast Enhancement with Collaborative Learning. Advances in Image and Video Processing, Volume 6 No 1, February (2018); pp: 1-12

processing. Segmentation is to divide brain images into several regions with different tissues like white matter, grey matter, and cerebrospinal fluid (CSF). The process of obtaining the regions of interest mainly includes brain normal tissue segmentation and abnormal lesion segmentation.

Image contrast [3-5] refers to the measurement of different levels of brightness between the brightest white and the darkest black in the light and dark regions of an image. The bigger the difference is, the higher the contrast is. The signal intensity of the magnetic resonance image is the result of a combination of T1, T2, proton density, flow, diffusion, perfusion, and other factors affecting signal emission in the voxel or the pixel. Compared with other medical imaging modalities, MRI has more parameters and more complicated design, which provide technology to improve the contrast between different tissues. For example, the human brain MRI is to detect the hydrogen signal of the tissue. Different tissues have different hydrogen contents, and can display high or low signal in different parameter choices, which gives a lot of choices on clinical applications. Therefore, MR image can provide richer information. However, since different contrast images can be produced for the same brain region, image segmentation is different on those different contrast images, even if the same segmentation method and parameters are used. High contrast MR brain image can increase segmentation accuracy in compared to segmentation on low contrast images. For this reason, image enhancement is necessary to be implemented before segmentation for obtaining accurate segmentation.

Spectral clustering [6] extracts the eigenvectors by eigenvalue decomposition of the data matrix to realize spectral mapping from high-dimensional complex structures to low-dimensional simple structures, and obtains a low-dimensional embedding of the original data in the new space. Lowdimensional embedding in the structure is more simple and obvious distribution. Compared with the traditional clustering algorithm, spectral clustering algorithm has obvious advantages. The algorithm can converge to the global optimum. In order to apply spectral clustering to the field of image segmentation, reduce the computational complexity and reduce the memory consumption of the algorithm, some methods have been studied and improved the spectral clustering algorithm. In the reference [7], mean shift algorithm [8] is combined with normalized cut algorithm [6] to classify the images by mean shift algorithm, resulting in a relatively large number of pure subclasses. Then by normalized cut algorithm is used for re-clustering these sub-class combination. The reference [9] proposed pixel sampling of the image by the Nyström method by sampling sampling pixel set and unsampled pixel set between weights matrix to obtain the original image weight matrix for generating approximation matrix, and the approximate weight matrix $W$ to approximately replace the eigenvectors of the original weight matrix. Keuchel et al [10] proposed the pixel based matrix weights vector sampling. The matrix of the singular value decomposition is used to approximate the original weight matrix, and then the eigenvectors of the original weight matrix are used to approximate the eigenvectors of the original weight matrix.

A novel MR brain image segmentation method is proposed in the paper. Low contrast MR brain image is enhanced with collaborative learning based image enhancement technique [11] at first. This image enhancement technique outperforms traditional image enhancement technique to make brain tissues contrast obviously. Then, a spectral clustering algorithm is implemented on the enhanced MR brain image for obtaining the segmentation results. The paper is organized as follows. The introduction and background of techniques are presented in the first two sections. The proposed method is given in the third section. Experimental result and conclusion are presented in the last two sections. 


\section{Background}

Image enhancement highlights useful information in an image according to a specific requirement. The purpose of image enhancement is to make the processed image more suitable for the visual characteristics of the human eye or for easy machine recognition. In medical imaging, remote sensing imaging, photography and other fields, image enhancement technology has a wide range of applications. At the same time, image enhancement can be used as a preprocessing algorithm for image processing algorithms such as object recognition, object tracking, feature matching, image fusion, and super-resolution reconstruction. Image enhancement improves the overall image and local contrast. Specifically, image enhancement algorithm not only improves the contrast of the whole image, but also enhances the local details of the image. Noise should be avoided during image enhancement. Noise in the image enhancement process is also enlarged, thus image quality is affected. Furthermore, enhanced image should have a good visual effect. Thus enhanced image should be consistent with the visual characteristics of the human eye. Image enhancement algorithm should have good real-time performance without obvious time delay.

Histogram equalization (HE) algorithm is the most basic image enhancement algorithm. Its principle is simple and easy to implement with good real-time. The histogram equalization algorithm achieves the purpose of increasing the dynamic range of the image and improves the contrast of the image by satisfying the probability density function (PDF) of the image grayscale in the form of approximately uniform distribution [12-14]. There are several improved algorithms based on histogram algorithm. They have their own characteristics. For example, Double Histogram Equalization (BBHE) algorithm, which solves the problem of uneven brightness in the enhanced image local area [15]. Exact histogram equalization [20] considers image enhancement as an ill-posed problem and replaces classical histogram equalization with direct verification of human visual models. Equal area double histogram equalization DSIHE algorithm and the two-dimensional spatial information entropy histogram equalization (SEHE) algorithm make the enhanced image that has the maximum information entropy and solve the problem of loss of image detail information caused by histogram equalization algorithm [16-19]. Maximum brightness double histogram equalization (MMBEBHE) algorithm ensures the least mean value error between the enhanced image and the original image [17]. LMHE algorithm based on logarithm function mapping makes the enhanced image more in line with the human eye visual characteristics [18].

Spectral clustering algorithm can be generalized as the following steps: (1) constructing a similarity matrix W; (2) constructing a Laplacian matrix L according to the similarity matrix W; (3) decomposing $\mathrm{L}$ matrix and selecting eigenvectors to produce a feature space; and (4) applying $\mathrm{K}$-means algorithm in feature space to output clustering results. The main advantages of spectral clustering algorithm only need the similarity matrix between data, so it is very effective for clustering sparse data. Traditional clustering algorithm such as K-Means is hard to achieve. In addition, due to the use of dimensionality reduction, the complexity in processing high-dimensional data clustering is better than the traditional clustering algorithm.

Spectral clustering algorithms have the advantage of a wide range of data with different applications. Clustering analysis based on pixels and their features is a very feasible for image segmentation. Therefore, this paper attempts to apply the spectral clustering algorithm to MRI image segmentation. The image clustering based on spectral clustering method is studied in this paper from the improvement of image contrast as a preliminary step to the application in MR image segmentation, especially in the segmentation of multiple inhomogeneous tissues of MR brain image. Finally, the experimental results are given and analyzed and compared. 


\section{Proposed Method}

The proposed framework is summarized in Figure 1. It is seen that the proposed method contains two steps. Original MR image is fed into image enhancement by the collaborative learning based technology [11]. Different tissue regions of enhanced image are easy to be divided into segments. It is then segmented by spectral clustering algorithm for producing the final segmentation result. Image enhancement and segmentation are sequentially combined to form the proposed method.

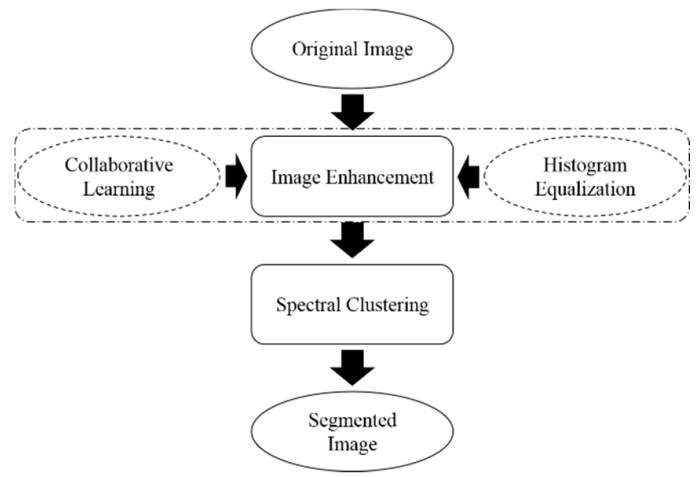

Figure 1. Framework of the 2-step MR image segmentation method.

The proposed image enhancement algorithm comes from collaborative learning of knowledge creation community [11]. The use of collaborative learning helps determine the final gray level of each pixel from multiple perspectives. It does not limit the gray level of each pixel in the context of the local window. This section describes the strategy for setting the gray level of each pixel based on local and global information. Each local window of the original image is randomly sampled for local histogram equalization. Note that both of each local window's center and size are randomly sampled, so that details of local information can be detected and identified. Integrated enhancement method refers to a combination of multiple image enhancers to obtain better results. The integrated model of multiple enhancement local windows has a more generalization ability. This is similar to learning ensemble method. The idea of learning ensemble is to integrate several single classifiers to determine the final classification by some combination of the classification results of multiple classifiers in order to achieve more than a single classifier performance. If a single classifier is compared to a single decision maker, the ensemble approach of learning is equivalent to multiple decision makers which make a joint decision. For training multiple models, the voting method may be used for the classification problem. The category with the largest number of votes may be selected as the final classification. For the regression problem, the mean value may be used as the final result. In this image enhancement technique, final grey values of pixels are calculated by averaging the mean values of multiple local enhanced window images. MR image are enhanced by this algorithm to differentiate tissue regions obviously. More details of the collaborative learning based image enhancement can be seen in the reference [11].

After MR image is enhanced, a spectral clustering algorithm is applied on enhanced image for segmentation, as shown in Figure 1. For the enhanced MR image $I_{E}$, all pixel values are given by $P=$ $\left\{p_{1}, p_{2}, \ldots, p_{n}\right\}$, which is divided into $C$ classes corresponding to $C$ homogeneous regions in final segmentation result. A similarity matrix is calculated as

$$
A_{i j}= \begin{cases}e^{-d^{2}\left(p_{i} \cdot p_{j}\right) / \sigma^{2}}, i \neq j \\ 0 & , i=j\end{cases}
$$


, where $d\left(p_{i} \cdot p_{j}\right)$ represents distance between two pixels and $\sigma$ is a scale parameter. Then, a Laplacian matrix is constructed by

$$
L=D^{-1 / 2} A D^{-1 / 2}
$$

, where $D$ is a diagonal matrix with $D_{i i} \sum_{j=1}^{n} A_{i j}$. The $C$ largest eigenvalues of the Laplacian matrix $L$. These $C$ largest eigenvalues are corresponding to $\lambda_{1} \geq \lambda_{2} \geq \cdots \geq \lambda_{C}$. The corresponding eigenvectors $x_{1}, x_{2}, \ldots, x_{C}$ are used to build a matrix $X=\left[x_{1}, x_{2}, \ldots, x_{C}\right]$. Each row of the matrix $X$ is normalized as follows.

$$
Y_{i j}=X_{i j} /\left(\sum_{j} x_{i j}^{2}\right)^{1 / 2}
$$

Each row of the matrix $Y$ is considered as a data point. The $k$-means clustering is applied data points $y_{1}, y_{2}, \ldots, y_{C}$ to obtain the final $C$ homogeneous regions of final segmentation result.

If the final clustering dimension is very high, the spectral clustering will not run at the very best speed and the final clustering result will not be good due to the insufficient dimensionality reduction. In addition, the clustering effect depends on the similarity matrix, and the final clustering results obtained by different similarity matrix may be very different in the final segmentation result.

\section{Experimental Results}

Two MR brain images are used for performance evaluation. Both MR images are fully sampled and reconstructed by fast Fourier transform. The first brain image is T1 weighted as shown in Figure 2 . Contrast between tissues of white matter, grey matter, and CSF is low in the T1 weighted brain image. Spectral clustering algorithm is applied on original MR brain image with low contrast. It is seen that boundaries between white matter and grey matter cannot be identified, so that both tissues are not able to be separated and segmented. Both of exact histogram equalization based image enhancement and the collaborative learning based image enhancement have been used for enhancing original T1 weighted MR brain image. Contrast is enhanced among white matter, grey matter, and CSF tissues. The collaborative learning based image enhancement has better performance than exact histogram equalization [20] enhanced image. After segmentation of spectral clustering, exact histogram equalization enhanced image cannot be segmented accurately at the bottom of the image. It is seen that contrast between white matter and grey matter is low. Therefore, right boundaries is difficult to be identified and segmentation is inaccurate. On the other hand, segmentation on collaborative learning enhanced image is more accurate than segmentations of original image and exact histogram equalization enhanced image.

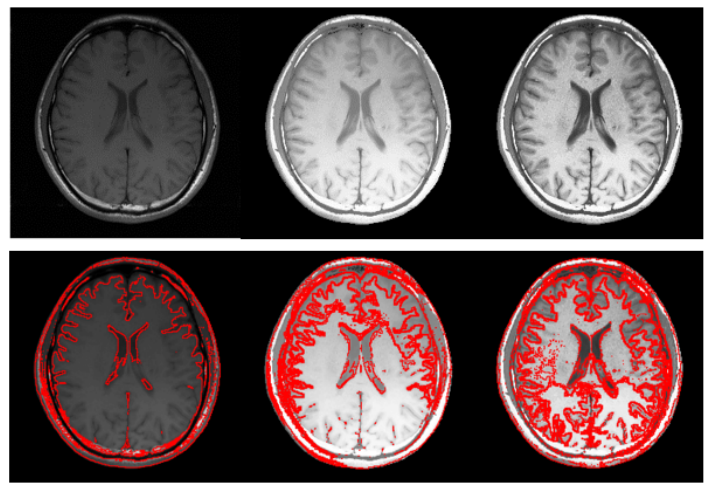

Figure 2. Three images on the first row presents T1 weighted MR brain image (left) with exact histogram equalization based image enhancement (middle) and collaborative learning based image enhancement

(right). Spectral clustering based image segmentation is applied on three images of the first row. T1 weighted MR brain image segmentation (left), exact histogram equalization enhanced image. 
Yuchou Chang; MR Image Segmentation Based on Contrast Enhancement with Collaborative Learning. Advances in Image and Video Processing, Volume 6 No 1, February (2018); pp: 1-12

The second brain image is T12 weighted as shown in Figure 3. Contrast between tissues of white matter, grey matter, and CSF is also low in the T2 weighted brain image. Spectral clustering algorithm is applied on original MR brain image with low contrast. It is seen that boundaries between white matter and grey matter is hard to be identified, so that both tissues are not separated and segmented accurately. Both of exact histogram equalization based image enhancement and the collaborative learning based image enhancement have been used for enhancing original T2 weighted MR brain image. Contrast is enhanced among white matter, grey matter, and CSF tissues. The collaborative learning based image enhancement has also better performance than exact histogram equalization enhanced image. After segmentation of spectral clustering, exact histogram equalization enhanced image cannot be segmented accurately, which is similar to T1 weighted image. It is seen that contrast between white matter and grey matter is low. Therefore, right boundaries is difficult to be identified and segmentation is inaccurate. However, segmentation on collaborative learning enhanced image is more accurate than segmentations of original image and exact histogram equalization enhanced image.
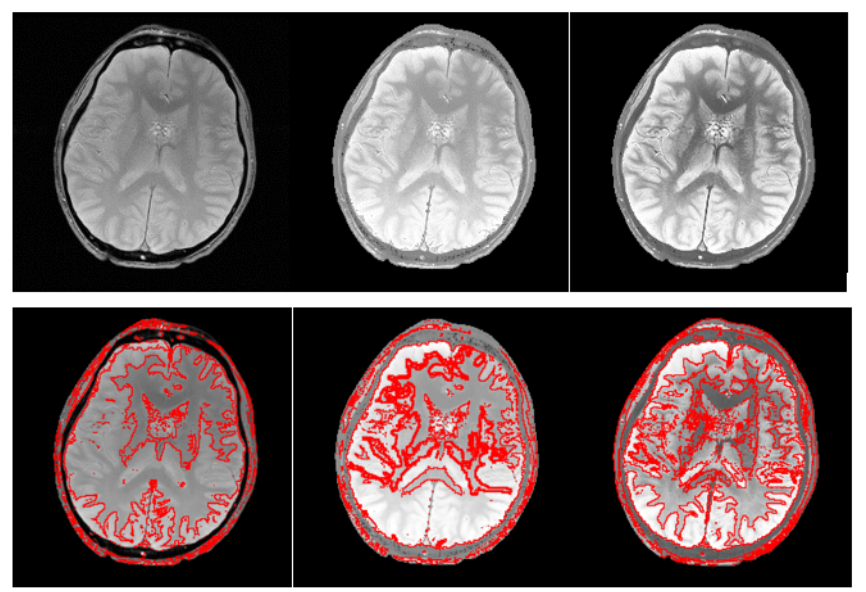

Figure 3. Three images on the first row presents T2 weighted MR brain image (left) with exact histogram equalization based image enhancement (middle) and collaborative learning based image enhancement

(right). Spectral clustering based image segmentation is applied on three images of the first row. T1 weighted MR brain image segmentation (left), exact histogram equalization enhanced image segmentation (middle), and the collaborative learning enhanced image segmentation (right) are presented on the second row.

In spectral clustering image segmentation algorithm, the construction of similarity matrix is the key to the proposed segmentation method. The similarity matrix affects the segmentation result directly. However, if the parameters involved in the construction of similarity matrix are not defined properly, inaccurate segmentation results may be produced. Massive data operations leading to the problem of low segmentation efficiency remains to be further resolved.

\section{Conclusion}

In summary, a MR image segmentation method is proposed in the paper. Since MR image contrast is determined by multiple factors during imaging process, low contrast image usually causes inaccurate segmentation result. The proposed method is composed of two steps: preliminary image enhancement and spectral clustering of different types of tissues. Enhanced image based segmentation achieve higher accuracy in compared to segmentation without pixel value enhancement. Experimental results of several MR images illustrate that the proposed segmentation method is able to divide inhomogeneous tissue regions accurately. 


\section{REFERENCES}

[1] Haacke, E.M., et al., Magnetic resonance imaging: physical principles and sequence design. Wiley-Liss, 1999.

[2] El-Baz, A., et al., Biomedical image segmentation: advances and trends. CRC Press, 2016.

[3] Stark J.A., Adaptive image contrast enhancement using generalizations of histogram equalization. Image Processing, IEEE Transactions on, 2000. 9(5): p. 889-896.

[4] Ibrahim, H., et al., Brightness preserving dynamic histogram equalization for image contrast enhancement. Consumer Electronics, IEEE Transactions on, 2007. 53(4): p. 1752-1758.

[5] Jen, T.C., Image contrast enhancement based on intensity-pair distribution. Image Processing, International Conference on, 2005.

[6] Tao, J., et al., Normalized cuts and image segmentation. Pattern Analysis and Machine Intelligence, IEEE Transactions on, 2000. 22(8): p. 888-905.

[7] Tao, V., et al., Color image segmentation based on mean shift and normalized cuts. Systems, Man, and Cybernetics - Part B: Cybernetics, IEEE Transactions on, 2007. 37(5): p. 1382-1388.

[8] Comaniciu, D., et al., Mean shift: a robust approach toward feature space analysis. Pattern Analysis and Machine Intelligence, IEEE Transactions on, 2002. 24(5): p. 603-619.

[9] Fowlkes C., et al., Spectral grouping using the Nystrom method. Pattern Analysis and Machine Intelligence, IEEE Transactions on, 2004. 26(2): p. 214-225.

[10] Keuchel J., et at., Efficient graph cuts for unsupervised image segmentation using probabilistic sampling and SVD-based approaximation. Statistical and Computational theories of Vision, Computer Vision, International Conference on, 2003.

[11] Chang, Y., et al., Using collaborative learning for image contrast enhancement. Pattern Recognition, International Conference on, 2008.

[12] Zimmerman, J.B., An evaluation of the effectiveness of adaptive histogram equalization for contrast enhancement. Medical Imaging, IEEE Transactions on, 1988. 7(4): p. 304-312.

[13] Wang, Q., et al., Fast image/video contrast enhancement based on weighted threshold histogram equalization. Consumer Electronics, IEEE Transactions on, 2007. 53(2): p. 757-764.

[14] Yang, S., et al., Constrast enhancement using histogram equalization with bin underflow and bin overflow. Image Processing, International Conference on, 2003.

[15] Kim, Y., Contrast enhancement using brightness preserving bi-histogram equalization. Consumer Electronics, IEEE Transactions on, 2007. 43(1): p. 1-8.

[16] Wang, Y., Image enhancement based on equal area dualistic sub-image histogram equalization method. Consumer Electronics, IEEE Transactions on, 2007. 45(1): p. 68-75.

[17] Chen, S., et al. Minimum mean brightness error bi-histogram equalization in contrast enhancement. Consumer Electronics, IEEE Transactions on, 2003. 49(4): p. 1310-1319. 
Yuchou Chang; MR Image Segmentation Based on Contrast Enhancement with Collaborative Learning. Advances in Image and Video Processing, Volume 6 No 1, February (2018); pp: 1-12

[18] Kim, W.K., et al., Contrast enhancement using histogram equalization based on logarithmic mapping. Optical Engineering, 2012. 51(6): p. 1-10.

[19] Celik, T., Spatial entropy-based global and local image contrast enhancement. Image Processing, IEEE Transactions on, 2014. 23(12): p. 5298-5308.

[20] Coltuc, D., et al., Exact histogram specification. Image Processing, IEEE Transactions on, 2006. 15(5): p. 1143-1152. 